\title{
Hallazgos de anticuerpos contra Leptospira sp., serovariedades Panama, Lai, Australis, Shermani y Patoc, en un grupo de monos rhesus (Macaca mulatta) en condiciones de cautiverio
}

\author{
Antibodies findings against Leptospira sp., of the serovars Panama, Lai, Australis, Shermani, \\ and Patoc, in a group of rhesus monkeys (Macaca mulatta) in conditions of captivity \\ A Ibáñez-Contreras ${ }^{\mathrm{a}^{*}}$, B Hernández-Godínez ${ }^{\mathrm{a}}$, JI Torres- Barranca ${ }^{\mathrm{b}}$, P Meléndez-Vélez \\ aUnidad de Primates no humanos del Centro de Investigación Proyecto CAMINA A.C., México DF. \\ bLaboratorio de Leptospira, de la Universidad Autónoma Metropolitana. Unidad Xochimilco, México DF.
}

\section{SUMMARY}

\begin{abstract}
During the last decade leptospirosis has emerged as a worldwide and very important infectious disease, affecting different environments from urban to rural, with carriers usually being wild or domestic animals. The objective of this study was to evaluate the presence of antibodies against Leptospira in rhesus monkeys (Macaca mulatta) in a Research Center. Fifty nine non-human primates of different physiological stages were studied. The animals were held in groups and in individual cages. Blood samples were obtained from the saphenous vein to determine the presence of antibodies through the microscopic agglutination test (MA), using twenty five serovars of Leptospira as antigens. It was observed that 18 out of the 59 monkeys (30.50\%) reacted against one or more serovars of Leptospira. In this study, antibodies of 4 serovars of Leptospira, (Panama, Lai, Australis, and Shermani) were detected. All 4 serovars were considered pathogenic for different mammals, including human beings, and eleven of the samples also reacted against the Patoc serovar, which is considered non pathogen and saprophyte. None of the studied animals that had a positive result against Leptospira presented any clinical signs compatible with leptospirosis.
\end{abstract}

Palabras clave: leptospirosis, Macaca mulatta, aglutinación microscópica, serovariedades, zoonosis.

Key word: leptospirosis, Macaca mulatta, microscopic agglutination test, serovars, zoonosis.

\section{INTRODUCCIÓN}

La leptospirosis es una zoonosis ampliamente distribuida que afecta a los animales domésticos y silvestres; en el caso de los humanos, ésta aparece como último eslabón de la cadena epidemiológica (Scarcelli y col 2003). La infección por lo regular se adquiere a temprana edad, por lo que la excreción urinaria de la leptospirosis aumenta conforme se incrementa la edad del animal (Levett 2001). Los signos más severos son observados en animales jóvenes, presentándose pirexia, anorexia, anemia hemolítica aguda, hemoglobinuria, ictericia y septicemia; formas severas terminan en muerte o convalecencias prolongadas. Los animales infectados de manera crónica presentan daños renales y hepáticos; en esta fase la infección puede resultar en abortos, muerte del neonato o nacimientos prematuros y débiles (Thiermann 1992, Rodríguez 2004)

La incidencia de la adquisición natural de leptospirosis en los primates no humanos es poco común, aun cuando experimentalmente se ha estudiado esta infección

Aceptado: 21.10.2009.

* Av. Tlalpan \# 4430, Col. Toriello Guerra, C.P. 14080 México, DF, México; Ibanez.alejandra@hotmail.com, Jany2104@hotmail.com. en una gran variedad de monos (Bennet y col 1995). Los primates no humanos pueden ser vectores naturales y pueden plantear un riesgo para su medio ambiente (Scarcelli y col 2003). Pereira y col (2005) inocularon Leptospira interrogans serovariedad Copenhageni en Callithrix jacchus reportando la presencia de hemorragias intraalveolares comparables a las vistas en las formas severas de leptospirosis humanas, con la presencia de áreas hemorrágicas extensivas e irregulares principalmente en el parénquima pulmonar, así como necrosis hemorrágica en el tejido musculoesquelético. De igual manera, Perolat y col (1992) confirman la alta susceptibilidad que presentan los Callithrix jacchus a la leptospirosis, serovariedad Icterohaemorrhagiae y la probable susceptibilidad a otros serogrupos. Bennet y col (1995) reportan la enfermedad clínica en monos del género Sanguinus, caracterizada por letargo, ictericia, disnea, hemorragias en la boca y la muerte.

En el caso de Cercopithecus aethiops infectados con L. borgpetersenii serovariedad Hardjo, Balcanica y Tarassovi, Hambleton y col (1990) reportan la ausencia de signos clínicos sugestivos de leptospirosis, pero se observó el incremento en la química sanguínea de la deshidrogenasa láctica (DHL), aspartato aminotransferasa (AST), alaninoaminotransferasa (ALT) y creatininkinasa (CK), así como la disminución de los niveles de hierro en sangre en 
algunos casos, acompañada por lesiones histopatológicas en algunos órganos.

Por otro lado, Palmer y col (1987) inocularon experimentalmente dos serotipos de L. interrogans serovariedad Hardjo en la especie Cercopithecus aethiops y al igual que Hambleton y col (1990) no se observó ninguna signología de la enfermedad clínica severa. No se encontró ninguna lesión macroscópica significativa en los tejidos, pero existió el aislamiento de la Leptospira a partir de sangre, orina y riñón de éstos.

En el caso de Kessler y Everard (1988) evaluaron la presencia de leptospirosis en una colonia de monos Macaca mulatta en condiciones de semilibertad en Cayo Santiago, hallando que la serovariedad con mayor prevalencia fue Icterohaemorrhagiae. Los resultados serológicos así como la excelente condición clínica, la baja mortalidad y los altos índices reproductivos indican que la leptospirosis no es un problema de salud en esta colonia. El objetivo de este trabajo es evaluar la presencia de anticuerpos contra Leptospira en monos rhesus (Macaca mulatta) en condiciones de bioterio.

\section{MATERIAL Y MÉTODOS}

La investigación se realizó de acuerdo a la Norma Oficial Mexicana NOM-062-ZOO-1999 (Especificaciones técnicas para la producción, cuidado y uso de los animales de laboratorio) y fue aprobada por el Comité Interno de Cuidado y Uso de Animales de Laboratorio (CICUAL) y por las comisiones de Ética e Investigación del Centro de Investigación Proyecto CAMINA, A.C.

Se utilizaron 59 primates no humanos del género Macaca mulatta alojados bajo condiciones de cautiverio, 38 hembras y 21 machos, divididos en cinco grupos, de 0,1 a 3,1 años, de 3,1 a 6,1 años, de 6,1 a 9,1 años, de 9,1 a 12,1 años, de 12,1 a 27,1 años de edad. Estos se encontraban en jaulas grupales con muros de azulejo, malla ciclónica (permitiendo el contacto con posibles vectores) y pisos de cemento con cubierta plastificada, a una temperatura ambiente de 20 a $25^{\circ} \mathrm{C}$. Los 59 animales son alimentados de acuerdo al $4 \%$ de su peso con alimento alto en proteína Monkey Chow 5045 Purina ${ }^{\circledR}, 25 \%$ de proteína tres veces al día y agua ad libitum.

Se extrajo una muestra de sangre de la vena safena para la determinación de anticuerpos, para lo cual los individuos fueron previamente sedados con TiletaminaZolacepam (Zoletil®) en una dosificación de $4 \mathrm{mg} / \mathrm{kg}$, por vía intramuscular (Booker y col 1982).

Posteriormente se valoraron los títulos de anticuerpos producidos a partir de los sueros obtenidos empleando la Técnica de Aglutinación Microscópica (TAM) bajo los estándares de la Organización Panamericana de la Salud (OPS), de la Organización Mundial de la Salud (OMS) y de la Organización Internacional de Epizootias (OIE) (Myers 1985, OIE 1996). Se realizaron diluciones dobles de suero con PBS, iniciando con una dilución 1:10. A cada dilución del suero se agregaron 0,05 ml de antígeno, obteniendo una dilución final de 1:20 y, a partir de ésta, se realizaron diluciones dobles hasta llegar a 1:80. Las placas se incubaron a temperatura ambiente y en condiciones de humedad por una hora (Smith y col 1994). Como antígenos se utilizaron 25 serovariedades de Leptospira.

Para la manutención y preparación de los antígenos, las serovariedades se cultivaron en medio de Cox modificado, al cual se agregó $10 \%$ de suero de conejo estéril y descomplementado con calor. Los cultivos se incubaron a $29 \pm 1{ }^{\circ} \mathrm{C}$ durante 7 días y su crecimiento se determinó por observación en un microscopio de campo oscuro (120x) marca Olympus CX41®. Los títulos de anticuerpos se determinaron en la dilución más alta del suero donde se observó el 50\% o más de aglutinación. La técnica fue realizada en el Laboratorio de Leptospira de la Universidad Autónoma Metropolitana-Xochimilco.

\section{RESULTADOS Y DISCUSIÓN}

Existen pocas investigaciones en donde se reporten cambios y signos clínicos que presentan los primates no humanos infectados con alguna serovariedad de Leptospira ya sea de forma natural o experimental. La infección de monos por leptospirosis en un medio natural es un acontecimiento inusual, pero se ha encontrado en exámenes serológicos o bajo condiciones excepcionales en cautiverio.

Se ha descrito que las diversas especies de monos así como las distintas serovariedades de Leptospira producen variables en los resultados obtenidos, lo que dificulta obtener conclusiones firmes (Pereira y col 2005).

Otros autores han descrito la presencia de anticuerpos contra Leptospira de las siguientes serovariedades; Copenhageni, Icterohaemorrhagiae, Hardjo, Balcanica y Tarassovi (Bennet y col 1995, Hambleton y col 1980, Kessler y Everard, 1988, Palmer y col 1987, Pereira y col 2005, Perolat y col 1992), mientras que en este trabajo se determinaron anticuerpos contra cuatro serovariedades de Leptospira, Panama, Lai, Australis y Shermani, consideradas como patógenas para los diferentes mamíferos incluyendo el hombre y once de las muestras reaccionaron contra la serovariedad Patoc, considerada no patógena y saprofita en títulos que variaron de 1:20 a 1:80. Ninguno de los animales que resultaron positivos contra Leptospira presentó signos clínicos compatibles con leptospirosis. De igual forma se presentaron títulos positivos, observándose la presencia de anticuerpos contra Leptospira en 18 animales, existiendo 6 machos $(33,33 \%)$ y 12 hembras $(66,66 \%)$.

Por otro lado se observó la presencia de más de una serovariedad, reportándose en una hembra adulta la presencia de anticuerpos contra las serovariedades Patoc y Shermani, y en una hembra senil las serovariedades Lai y Shermani.

En el cuadro 1 se observa la cantidad de animales que presentaron anticuerpos contra Leptospira, así como las serovariedades más frecuentes en relación a la etapa fisiológica de los animales. Las serovariedades que más 
Cuadro 1. Presencia de las diferentes serovariedades de Leptospira por etapa fisiológica de los monos rhesus.

Presence of different Leptospira serovars by physiological stage of rhesus monkeys.

\begin{tabular}{lcl}
\hline Etapa fisiológica & Número de animales & Serovariedad \\
\hline $\begin{array}{l}\text { Neonatos e infantiles } \\
(0,1<3,1 \text { años })\end{array}$ & 1 & - Patoc \\
& & \\
$\begin{array}{l}\text { Infantiles y juveniles } \\
(3,1<6,1 \text { años })\end{array}$ & 1 & - Patoc \\
& 1 & - Lai \\
& 3 & - Panama \\
& 1 & - Australis \\
$\begin{array}{l}\text { Adultos jóvenes } \\
(6,1<9,1 \text { años })\end{array}$ & 3 & - Patoc \\
& & \\
Adultos & & \\
$(9,1<12,1$ años $)$ & 5 & - Patoc \\
& 1 & - Shermani \\
Seniles & & \\
$(12,1<27,1$ años $)$ & 1 & - Australis \\
& 1 & - Lai \\
& 1 & - Patoc \\
& 1 & - Shermani \\
\hline
\end{tabular}

se presentaron en los 18 animales positivos se observan en el cuadro 2, al igual que los títulos obtenidos de los animales serorreaccionantes.

En base a las diversas investigaciones existe una mayor sensibilidad a la Leptospira en los primates no humanos del Nuevo Mundo tales como los Callithrix jacchus y Callithrix kuhlii y en monos del género Sanguinus, de los cuales se ha reportado una signología similar a la padecida por los humanos (Baitchman y col 2006, Bennet y col 1995, Pereira y col 2005, Perolat y col 1992). A diferencia de los estudios realizados en primates del Viejo Mundo como Cercopithecus aethiops y Macaca mulatta, se observa que no existe ninguna respuesta desfavorable en la salud de éstos a la presencia de leptospirosis, indistintamente de la serovariedad adquirida (Hambleton y col 1980, Baulu y col 1987, Palmer y col 1987, Kessler y Everard, 1988).

Los títulos de anticuerpos determinados no se asociaron a un cuadro clínico sugestivo de leptospirosis en monos, determinándose un proceso de infección sin manifestaciones clínicas; por lo que al no existir contagio entre los monos y hacia el ser humano no existiría ningún riesgo de laborar con ellos. Los resultados sugieren que el mono puede ser hospedero accidental de las serovariedades determinadas al no existir un contagio o transmisión de la Leptospira entre su misma especie y hacia el humano.

Cuadro 2. Porcentaje de presencia por serovariedad de leptospirosis en monos rhesus.

Percentage of presence of leptospirosis serovar in rhesus monkeys.

\begin{tabular}{|c|c|c|c|c|c|}
\hline \multirow{2}{*}{ Serovariedad } & \multicolumn{4}{|c|}{ No. de monos positivos } & \multirow{2}{*}{ Porcentaje } \\
\hline & Tatuaje & Edad & Sexo & Titulación & \\
\hline Patoc & $\begin{array}{l}\text { F49 } \\
\text { F310 } \\
\text { F312 } \\
\text { F27 } \\
\text { F65 } \\
\text { F413 } \\
\text { F37 } \\
\text { F24 } \\
\text { F36 } \\
\text { F33 } \\
\text { F11 }\end{array}$ & $\begin{array}{r}1 \\
3 \\
5 \\
5 \\
6 \\
6 \\
7 \\
8 \\
8 \\
12 \\
23\end{array}$ & $\begin{array}{l}\text { M } \\
M \\
H \\
H \\
H \\
H \\
H \\
H \\
H \\
M \\
M\end{array}$ & $\begin{array}{l}1: 20 \\
1: 40 \\
1: 40 \\
1: 20 \\
1: 80 \\
1: 20 \\
1: 20 \\
1: 80 \\
1: 20 \\
1: 20 \\
1: 40\end{array}$ & $\begin{array}{l}61,11 \% \\
(11 \text { animales positivos }\end{array}$ \\
\hline Panama & $\begin{array}{l}\text { F32 } \\
\text { F51 } \\
\text { F62 }\end{array}$ & $\begin{array}{l}2 \\
2 \\
3\end{array}$ & $\begin{array}{l}\mathrm{H} \\
\mathrm{M} \\
\mathrm{M}\end{array}$ & $\begin{array}{l}1: 20 \\
1: 20 \\
1: 20\end{array}$ & $\begin{array}{l}16,66 \% \\
\text { ( } 3 \text { animales positivos) }\end{array}$ \\
\hline Lai & $\begin{array}{l}\text { F52 } \\
\text { F12 }\end{array}$ & $\begin{array}{r}2 \\
20\end{array}$ & $\begin{array}{l}\mathrm{H} \\
\mathrm{H}\end{array}$ & $\begin{array}{l}1: 20 \\
1: 20\end{array}$ & $\begin{array}{l}11,11 \% \\
\text { ( } 2 \text { animales positivos) }\end{array}$ \\
\hline Australis & $\begin{array}{l}\text { F53 } \\
\text { F13 }\end{array}$ & $\begin{array}{r}2 \\
20\end{array}$ & $\begin{array}{l}\mathrm{H} \\
\mathrm{H}\end{array}$ & $\begin{array}{l}1: 20 \\
1: 20\end{array}$ & $\begin{array}{l}11,11 \% \\
\text { ( } 2 \text { animales positivos) }\end{array}$ \\
\hline Shermani & $\begin{array}{l}\text { F37 } \\
\text { F12 }\end{array}$ & $\begin{array}{r}7 \\
20\end{array}$ & $\begin{array}{l}\mathrm{H} \\
\mathrm{H}\end{array}$ & $\begin{array}{l}1: 20 \\
1: 20\end{array}$ & $\begin{array}{l}11,11 \% \\
(2 \text { animales positivos) }\end{array}$ \\
\hline
\end{tabular}




\section{RESUMEN}

La leptospirosis ha emergido como una importante enfermedad infecciosa, lo que ha ocurrido en diferentes entornos desde urbanos hasta medios naturales; los portadores son animales salvajes o domésticos. El objetivo fue evaluar la presencia de anticuerpos contra Leptospira en monos rhesus en condiciones de bioterio. Se utilizaron 59 monos de diferentes etapas fisiológicas. Se determinaron anticuerpos contra Leptospira a partir de la prueba de aglutinación microscópica (TAM), utilizando como antígenos 25 serovariedades de Leptospira. Se observó que de los 59 animales, únicamente el 30,50\% reaccionaron contra una o más serovariedades de Leptospira. En este trabajo se determinaron anticuerpos contra cuatro serovariedades de Leptospira: Panama, Lai, Australis y Shermani, consideradas como patógenas, y once de las muestras reaccionaron contra la serovariedad Patoc, considerada no patógena y saprófita. Ninguno de los animales que resultaron positivos contra Leptospira presentó signos clínicos compatibles con leptospirosis.

\section{REFERENCIAS}

Baitchman EJ, PP Calle, SB James, MJ Linn, BL Raphael. 2006. Leptospirosis in Wied's marmosets (Callithrix kuhlii). J Zoo Wild Med 37, 182-185.

Baulu J, COR Everard, JD Everard. 1987. Leptospires in vervet monkeys (Cercopithecus aethiops sabaeus) on Barbados. J Wildl Dis 23, 60-66.

Bennet T, C Abee, R Henrickson. 1995. Nonhuman Primates in Biomedical Research: Biology and Management. Academic Press, San Diego, CA, USA.

Booker JL, HH Erickson, EL Fitzpatrick. 1982. Cardiodynamics in the rhesus macaque during dissociative anesthesia. Am J Vet Res 43, 671-676.

Eugster AK. 1996. Manual of standards for diagnostic tests and vaccines. $3^{\text {rd }}$ ed. Office International des Epizooties Paris, France, Pp 198-206.
Hambleton P, A Baskerville, RB Marshall, PW Harris-Smith, GD Adams. 1980. Metabolic sequelae of experimental Leptospirosis in grivet monkeys. Br J Exp Pathol 61, 16-21.

Kessler MJ, COR Everard. 1988. Leptospiral agglutinins in the Cayo Santiago macaques. Am J Primatol 14, 369-373.

Levett PN. 2001. Leptospirosis. Clin Microbiol Rev 14, 296-326.

Myers DM. 1985. Manual de métodos para el diagnóstico de laboratorio de la leptospirosis. Centro Panamericano de Zoonosis, OPS, OMS. Nota técnica $\mathrm{N}^{\mathrm{o}} 30$.

Palmer MF, SA Waitkins, RB Fitzgeorge, A Baskerville. 1987. Experimental infection of monkeys with Leptospira interrogans serovar Hardjo. Epidemiol Infect 98, 191-197.

Pereira MM, JJ Pereira Da Silva, M Alves Pinto, M França Da Silva, M Pelajo Machado, H Leonel Lenzi, RS Marchevsky. 2005. Experimental Leptospirosis In Marmoset Monkeys (Callithrix jacchus): A New Model For Studies Of Severe Pulmonary Leptospirosis. Am J Trop Med Hyg 72, 13-20.

Perolat P, JP Poingt, JC Vie, C Jouaneau, G Baranton, J Gysin. 1992. Occurrence of severe Leptospirosis in a breeding colony of squirrel monkeys. Am J Trop Med Hyg 46, 538-545.

Rodríguez, VR. 2004. Enfermedades de Importancia Económica en Producción Animal. McGraw Hill, México D.F, Pp 489-504.

Scarcelli ER, Ma Piatti, JD Luzes Fedullo, F Simon, M Vasconcellos Cardoso, V Castro, S Miyashiro, MÉ Genovez. 2003. Leptospira spp detection by Polymerase Chain Reaction (PCR) in clinical samples of captive black-capped capuchin monkey (Cebus apella). Braz J of Microbiol 34, 143-146.

Smith CR, PJ Ketterer, MR McGrowan, BG Corney. 1994. A review of laboratory techniques and their use in the diagnosis of Leptospira interrogans serovar Hardjo infection in the cattle. Aust Vet J 71, 290-293.

Thiermann AB. 1992. Leptospirosis una Importante Zoonosis en Producción Animal. National Animal Disease Center, Ames, Iowa, USA. 